\title{
Intelligentes Nesting in der Kreislaufwirtschaft zur Steigerung der Ressourceneffizienz
}

Philipp Kronenberg, Franz Wieck, Sebastian Weber, Manuel Löwer

Kreislaufwirtschaft ist ein Konzept, um den Rohstoff- und Ressourcenverbrauch wirksam zu reduzieren. Hierbei sollen bestehende Produkte und Ressourcen so lange wie möglich in wertschöpfenden Kreisläufen gehalten werden. Im Rahmen der Repurpose (Wiederverwendung) Strategie werden Bauteile und Ressourcen von ausrangierten Produkten in neue Produkte mit anderer Funktion wiederverwendet. Hierzu müssen Rohlinge für neue Produkte in Sekundärrohstoffen angeordnet und herausgetrennt werden. Bedingt durch den nicht standardisierten Zustand von Sekundärrohstoffen, wird ein intelligentes Nesting Verfahren eingesetzt, die Ressourceneffizienz zu verbessern. Der Einsatz von intelligentem Nesting im Rahmen der Kreislaufwirtschaft zeigt, dass der Einsatz von Sekundärrohstoffen gesteigert und die benötigte Energie für die nachfolgenden Fertigungsverfahren eingespart werden kann.

Keywords: Kreislaufwirtschaft, Nachhaltigkeit, Ressourceneffizienz, Sekundärrohstoffe, Nesting

\section{Einleitung und Motivation}

Der nachhaltige Umgang mit begrenzt vorhandenen Ressourcen ist gegenwärtig eine der größten Herausforderungen in Wissenschaft, Wirtschaft, Gesellschaft und Politik. Der Ressourcenverbrauch wird durch die Zunahme der Weltbevölkerung weiter steigen. Im Jahr 2017 sind 90 Milliarden Tonnen Rohstoffe, dreimal mehr als im Jahr 1970, der Natur entnommen worden (Stephan Lutter, Stefan Giljum, Burcu Gözet, Hanspeter Wieland, Christopher Manstein, 2018). Dieser Anstieg verursacht weltweite Umweltprobleme wie den Klimawandel, Bodendegradation und den Verlust an biologischer Vielfalt. Eine wesentliche Strategie, den Rohstoff- und Ressourcenverbrauch wirksam zu reduzieren, stellt das Konzept der Kreislaufwirtschaft (engl. Circular Economy, CE) dar.

Bei diesem Konzept sollen bestehende Produkte und Ressourcen so lange wie möglich geteilt, geleast, wiederverwendet, repariert, aufgearbeitet und recycelt werden. Durch diese ganzen Maßnahmen wird der Lebenszyklus von Produkten verlängert und somit 
die Ressourceneffizienz erhöht. Potting, Hekkert, Worrell und Hanemaaijer (2017) haben zehn der sogenannten R-Strategien anhand des Zirkularitätsgrades eingeteilt. Refuse, Rethink und Reduce fokussieren sich auf das Überdenken ganzer Produktsysteme und zielen auf einen disruptiven Wandel ab. Die Strategien Reuse, Repair, Refurbish, Remanufacture und Repurpose setzen an bestehenden Produktsystemen an und zielen darauf $a b$, deren Funktion zu erhalten, um die in den Produkten gebunden Ressourcen möglichst lange in der Nutzungsphase zu halten. Recycle und Recover bilden die dritte Kategorie der Strategien, die sich auf die Rückgewinnung von Ressourcen konzentrieren, die nicht mehr verwendet werden können.

Das im Rahmen dieses Beitrags erarbeitete Vorgehen bezieht sich auf die Strategie Repurpose. Laut Potting, Hekkert, Worrell, und Hanemaaijer (2017) werden bei dieser Strategie ausrangierte Produkte oder dessen Bauteile und Ressourcen in einem neuen Produkt mit anderer Funktion wiederverwendet. Hierbei behalten die aus einem ausrangierten Produkt verwendeten Materialien im Idealfall ihre ursprüngliche Qualität. Infolgedessen werden keine zusätzlichen natürlichen Ressourcen für die Herstellung von Materialien benötigt und ausrangierte Produkte werden nicht mehr zu Abfall.

\section{Zielsetzung der Untersuchung}

Der Aufsatz untersucht den Einfluss vom intelligenten Nesting auf die Ressourceneffizienz in Abhängigkeit des ausgenutzten Materials und der Prozessenergien. Das Konzept der Kreislaufwirtschaft beruht nicht nur auf der Materialeffizienz, sondern ist als übergeordnete Strategie in den jeweiligen Unternehmen verankert. So kann eine gezielte Positionierung von Rohlingen in Sekundärrohstoffen unter Berücksichtigung von multikriteriellen Faktoren neben einer Steigerung des Materialeinsatzes, Ressourcen (bspw. Energie und Wasser) in den nachfolgenden Fertigungsschritten einsparen. Im Rahmen dieses Beitrags soll an einem Beispiel der Werkzeug- und Schneidwarenindustrie gezeigt werden, welches Potenzial in diesem Ansatz steckt.

\section{Stand der Technik und Abgrenzung}

Nesting (auch Schachteln oder Verschachtelung) ist ein Begriff, der seit den 70er Jahren im Bereich der Fertigungstechnologien immer mehr an Bedeutung gewonnen hat (Michael Adamowicz, Antonio Albano,1976). Nesting bezeichnet die platzsparende Anordnung von Geometrien beim Ausschneiden, Verpacken oder Lagern. Das am häufigsten vorkommende Problem ist das sogenannte "strip-packing", bei dem eine Anzahl an unregelmäßigen Formen innerhalb einer rechteckigen Form platziert werden 
muss, so dass die Fläche bestmöglich genutzt werden kann. Wichtig ist, dass keine der Formen überlappen und so wenig Material wie möglich ungenutzt bleibt.

Klassische Beispiele hierfür sind die Positionierung von Geometrien auf flachem Rohmaterial wie z. B. Blech oder die Bekleidungsindustrie, in der Kleidungsstücke möglichst platzsparend auf einer Geweberolle angeordnet werden.

Obwohl sich der Begriff Nesting weitestgehend auf zweidimensionale Probleme bezieht, existieren viele Anwendungen im dreidimensionalen Bereich. Die platzsparende Positionierung von Objekten in einem vorgegebenen dreidimensionalen Raum ist eine Herausforderung in der additiven Fertigung und in der Logistikindustrie.

Der größte Unterschied zwischen der Verschachtelung von geschnittenen Bauteilen zu 3-Dimensionalen Nesting Problemen besteht darin, dass 3D-Teile in den meisten Fällen einen Querschnitt haben, der sich mit der Höhe ändert. Dies kann beim Aufbau zu Interferenzen zwischen benachbarten Teilen führen und erfordert somit deutlich mehr Rechenleistung, um ein solches Problem zu lösen.

\section{Nesting-Arten}

Im Wesentlichen lässt sich Nesting in 1-, 2- und 3-Dimensionale Anwendungen unterteilen, welche sich wiederum in ihrer Komplexität und in ihren Lösungsansätzen unterscheiden. Je nach Anwendung besitzen die Nestingarten verschiedene Optimierungsziele. Alle Nestingarten besitzen einen Designraum (engl. Designspace), Freiheitsgrade (engl. Degrees of Freedom), Randbedingungen (engl. Boundary Conditions), Komponenten (engl. Components) und Optimierungsziel (engl. Optimisation Target). Die Herausforderung besteht darin diese Parameter in einen mathematischen Zusammenhang zu überführen und hinsichtlich des Optimierungsziels die beste Variante zu ermitteln.

\section{Linear (1-dimensional)}

1D-Nesting ist das simpelste Verfahren aller Nesting Varianten. Es dient zur Unterstützung beim Erstellen von Schneidplänen für Halbzeuge wie beispielsweise Stangen, Profile, Träger oder Rohre. Durch den Einsatz von 1D-Nesting kann der Verschnitt und die Ressourceneffizienz gesteigert werden. Neben den herauszutrennenden Längen muss das Nesting zusätzlich die Schnittbreite der trennenden Fertigungsverfahren beim Schachteln berücksichtigen. Ein typisches Beispiel für das 1D-Nesting ist das Zersägen von Aluminium Profilen mit einer definierten Länge in kürzere Profilstücke unter Berücksichtigung einer festgelegten Bauteillänge, wobei der Verschnitt minimiert wird. 


\section{Plate (2-dimensional)}

Das 2D-Nesting ist ein Verfahren zum platzsparenden Anordnen von Geometrien auf Flächen. Die zu positionierenden Geometrien können dabei jede beliebige Form annehmen. Das Verfahren des 2D-Nesting ist bis heute Stand der Technik in der Blechverarbeitung. Als Arbeitsvorbereitung beim Laserschneiden werden auf Blechtafeln automatisiert die auszuschneidenden Geometrien positioniert, sodass möglichst wenig Material der Blechtafel ungenutzt bleibt. Neben der Materialeffizienz wird, wie beim 1D-Nesting, die Schnittfuge durch den gewählten Schneidprozess bei der Anordnung auf dem Belch berücksichtigt. Neben der Blechverarbeitung wird das Verfahren des 2D-Nesting in der Bekleidungsindustrie eingesetzt. Hierbei werden Teile von Kleidungsstücken auf Geweberollen angeordnet. Da die Breite dieser Rollen nicht variiert, ist das Ziel des Nesting die Länge des benötigten Stoffes zu reduzieren.

Neben der Materialeffizienz berücksichtigt das 2D-Nesting in der Lederindustrie für die Automobilindustrie ebenfalls die Anforderungen des nachfolgenden Fertigungsschrittes. Hierbei werden Fehlstellen auf dem Leder, wie Farbfehler oder Schäden, beim Nesting ausgelassen, sodass die Zuschnitte die Qualitätskriterien der nachfolgenden Fertigungsschritte einhalten können.

\section{Packing (3-dimensional)}

Das 3D-Nesting ist ein Verfahren für eine optimale Anordnung von Objekten in dreidimensionalen Räumen. Dabei können die Objekte selbst jedoch beliebig komplexe Geometrien annehmen. Dieses Verfahren wird hauptsächlich in der Logistikbranche oder der additiven Fertigung angewendet. Im Einsatzfeld der Logistik wird durch das Nesting Verpackungsmaterial und Lagervolumen eingespart. Des Weiteren wird 3DNesting dafür eingesetzt die Beladung von Schiffen oder Lastkraftwagen zu planen und zu optimieren. Im Bereich der additiven Fertigung wird das Nesting dazu verwendet, die zu fertigenden Bauteile in den zur Verfügung stehenden Bauraum so anzuordnen, dass eine hohe Auslastung des Bauraumes erreicht wird.

\section{Steigerung der Ressourcen Effizienz durch intelligentes Nesting}

\section{Problemstellung}

Die laut Potting, Hekkert, Worrell, und Hanemaaijer (2017) in der Repurpose Strategie wiederverwendeten Ressourcen werden als Sekundärrohstoffe bezeichnet. Im Gegen- 
satz zu Primärrohstoffen, die aus der Natur gewonnen sind, werden Sekundärrohstoffe zum zweiten oder wiederholten Male genutzt. Je nach Material, Zustand, Beschaffenheit, technischen Verfahren und Aufwand können verschiedene Rohstoffe (Metalle, Glas, Polymere, Holzfasern) unterschiedlich oft als Sekundärrohstoff wiederaufbereitet und verwendet werden. Dabei kann die Aufbereitung durch Zerkleinern, Trennen oder andere Verfahren erheblich mehr Energie erfordern als die Verwendung von Primärrohstoffen. Durch neue bzw. verbesserte Verfahren kann der Anteil an verwendeten Sekundärrohstoffen deutlich erhöht und Energie eingespart werden (Hermani, C., 2018). Das in diesem Beitrag vorgestellte Vorgehen fokussiert sich dabei darauf, dass durch intelligentes Nesting Sekundärrohstoffe verwendet und darüber hinaus die benötigten Energien für die nachfolgenden Fertigungsverfahren minimiert werden. Hierbei besteht die Herausforderung, dass die Sekundärrohstoffe keinen standardisierten Zustand aufweisen. Zu berücksichtigen sind unter anderem Verschmutzungen, unterschiedlich starke Abnutzung oder Inhomogenitäten der Produkte und Rohstoffe. Weiterhin wird untersucht, wie die Lage der zu schachtelnen Bauteile den Ressourcenverbrauch und den Ausnutzungsgrad des Sekundärrohstoffs beeinflussen. In dem hier dargestellten Beispiel aus der Werkzeug- und Schneidwarenindustrie sollen die Herausforderungen und positiven Effekte des intelligenten Nestings dargestellt werden.

\section{Praxisbeispiel in der Werkzeug- und Schneidwarenindustrie}

Als Sekundärrohstoff werden verschlissene Maschinenkreismesser untersucht. Das Potential für die Weiterverarbeitung der Maschinenkreismesser liegt darin, dass der Verschleiß nur an der Schnittkante aufritt (Durchmesser um 10\% geringer), der homogene und aufwendig hergestellte Werkzeugstahl (6kg Werkzeugstahl pro Messer) und die jährlich anfallende Stückzahl von über 100.000 (Stk/Jahr). Da bereits für die Herstellung des hochlegierten Werkzeugstahls und die Produktion der Messer viel Energie benötigt worden ist, soll der Werkzeugstahl im Sinne der Kreislaufwirtschaft weiter in wertschöpfenden Prozessen gehalten werden. Durch das Anwenden der Repurpose Strategie sollen aus dem Maschinenkreismesser Rohlinge für andere Schneidwaren und Werkzeuge herausgetrennt werden (siehe Abbildung 1). An diesem Punkt wird das intelligente Nesting eingesetzt, dass mit den zur Verfügung stehenden Informationen wie das Volumen, die Geometrie, das Material und anderen Daten über die Sekundärrohstoffe, die optimale Anordnung der Rohlinge vornimmt. Dabei zwei Aspekte berücksichtigt werden:

1. Eine verschnittarme Entnahme und hohe Materialausnutzung

2. Berücksichtigung der nachfolgenden Fertigungsverfahren 


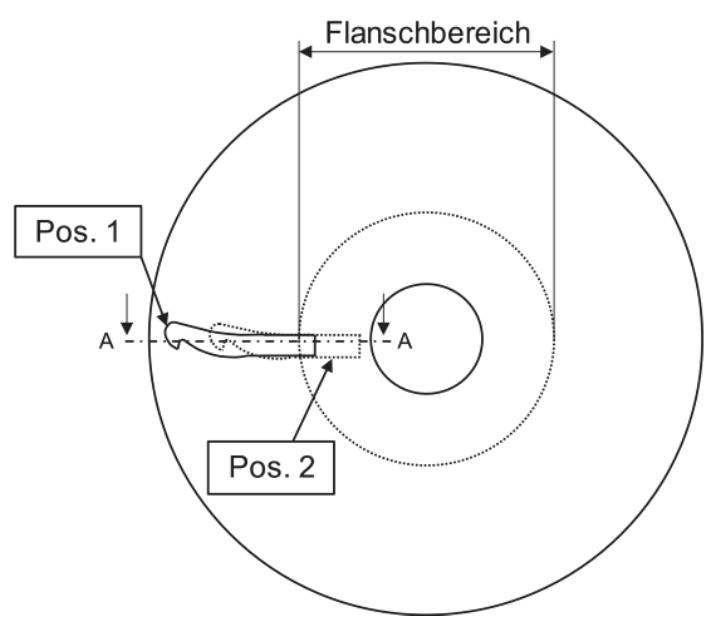

Abbildung 1: Schematische Darstellung eines Maschinenkreismessers mit zwei Positionen eines Messerrohlings.

Da es sich bei dem hier zur Verfügung stehenden Sekundärrohstoff nicht um Blechtafeln handelt, die üblicherweise in der Blechverarbeitung eingesetzt werden, muss das neuartige Nesting ebenfalls Materialstärken Änderungen berücksichtigen. In Abbildung 2 sind Schnittdarstellungen mit dieser Problematik dargestellt. Der innere Bereich, auch Flanschbereich genannt, weist eine durchgängige Materialstärke von $5 \mathrm{~mm}$ auf. Durch die angeschliffene Wate des Messers reduziert sich die Materialstärke bis zum Rand auf unter $1 \mathrm{~mm}$.

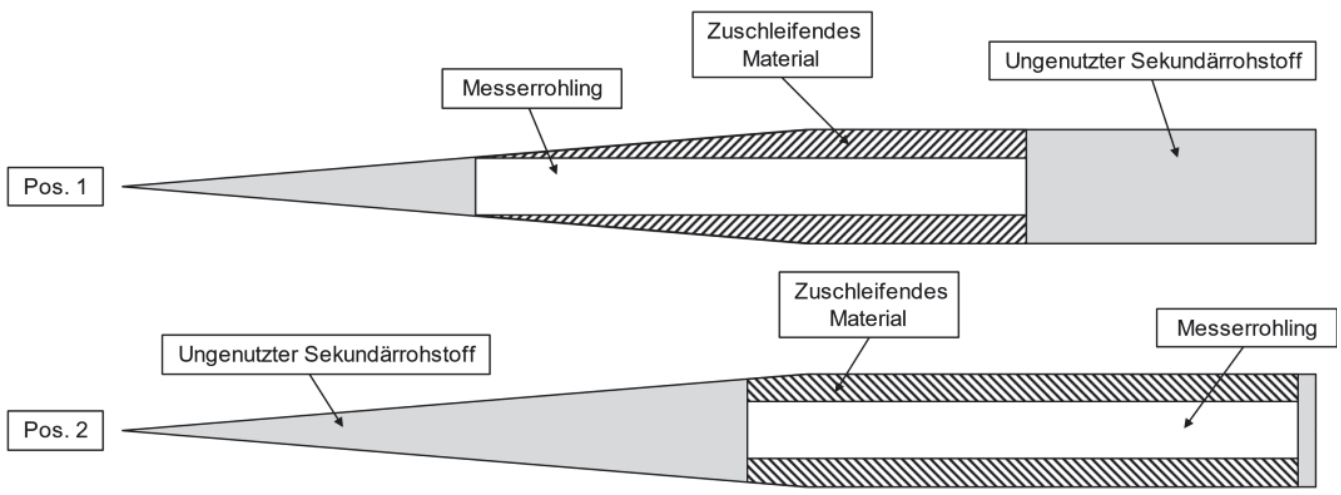

Abbildung 2: Schematische Schnittdarstellung des Maschinenkreismesser mit zwei verschiedenen Positionen des Messerrohlings. 
In Abbildung 2 sind zwei verschiedene Positionierungen eines Rohlings der Werkzeugindustrie dargestellt. An der Position 1 ist der Rohling so positioniert, dass die finale Materialstärke von $2 \mathrm{~mm}$ des Rohlings am linken Rand vorliegt. Das überschüssige Material, dass durch Schleifen in nachfolgenden Fertigungsschritten entfernt werden muss, ist schraffiert dargestellt. Bei dieser Variante wird 47,19 g Material weggeschliffen, wofür 2,55 MJ Energie verbraucht wird (CES Selector, 2019). Dem gegenüber steht die zweite Positionierung, bei der der Rohling hauptsächlich im Flanschbereich liegt. Die nachzubearbeitende Fläche ist in dieser Variante ebenfalls schraffiert dargestellt. Deutlich zu sehen ist, dass über den ganzen Querschnitt Material weggeschliffen werden muss, dass einer Menge von 64,67 g entspricht. Hierfür wird 3,49 MJ Energie benötigt. Gegenüber der linken Variante müsste also 37,01 \% mehr Energie aufgewendet werden.

\section{Zusammenfassung und Diskussion}

Das im Rahmen dieses Beitrags vorgestellte Verfahren, intelligentes Nesting als Werkzeug im Rahmen einer Kreislaufwirtschaft zu verwenden hat gezeigt, dass durch den Einsatz die Nutzung von Sekundärrohstoffen gesteigert und der Energieverbrauch der nachfolgenden Fertigungsverfahren reduziert werden kann. Das Verfahren des Nestings ist bereits in vielen anderen Branchen Stand der Technik, sodass die Hürde in der Umsetzung nicht an der Technologie an sich liegt, sondern lediglich in der Bereitstellung der Informationen über die Sekundärrohstoffe. Hierzu müssen Datenbanksysteme entwickelt werden, die Informationen über Sekundärrohstoffe und den daraus zu fertigenden Produkten beinhalten. In früheren Arbeiten haben Wieck, Schmidtseifer, Kronenberg, Deuerler und Löwer (2020) bereits die Relevanz von diesen Datenbanken identifiziert. In nachfolgenden Arbeiten müssen diese automatisierten Nestings weiter erprobt und durch Informationen der nachfolgenden Fertigungsverfahren weiter ergänzt werden. Des Weiteren muss das Verfahren auf andere Produkte und Industrien ausgeweitet werden.

\section{Finanzierung}

Die vorliegende Arbeit ist im Rahmen eines BMBF-Verbundprojektes "ReziProK: COT CIRCLE OF TOOLS: Entwicklung und Erprobung von Demonstratoren im Kontext einer zirkulären Wertschöpfung von Werkzeugstählen." unter dem Kennzeichen 033R230A entstanden. 


\section{Literaturverzeichnis}

Stephan Lutter, Stefan Giljum, Burcu Gözet, Hanspeter Wieland, Christopher Manstein (2018): Die Nutzung natürlicher Ressourcen, Bericht für Deutschland 2018: Bundesumweltamt

Potting, J., Hekkert, M., Worrell, E., \& Hanemaaijer, A. (2017): Circular Economy: Measuring Innovation in the Product Chain., PBL Netherlands Environmental Assessment Agency, Hrsg.

Michael Adamowicz, Antonio Albano (1976): Nesting two-dimensional shapes in rectangular moduls

Hermani, C. (2018): Rohstoffpolitik 2.0, Für eine Umwelt- und Klimagerechte Ressourcennutzung: Deutscher Naturschutzring Dachverband der deutschen Natur-, Tier- und Umweltschutzorgaisationen (DNR) e.V.

CES Selector 2019 (Granta Design Limited, 2019)

Wieck, F., Schmidtseifer, N., Kronenberg, P., Deuerler, F., Löwer, M. (2020): Tagungsband: 18. Gemeinsames Kolloquium Konstruktionstechnik: Nachhaltigkeit messbar machen - eine neue Kennzahl für Repurpose-Prozesse, 321 - 332. DuisburgEssen Publications online

\section{Kontakt}

Philipp Kronenberg, M. Sc.

Franz Wieck, M. Sc. RWTH

Univ.-Prof. Dr.-Ing. Manuel Löwer

Bergische Universität Wuppertal

Lehrstuhl für Neue Fertigungsverfahren und Werkstoffe

42651 Solingen

www.fuw.uni-wuppertal.de

Prof. Dr.-Ing. Sebastian Weber

Ruhr-Universität Bochum

Lehrstuhl Werkstofftechnik

44801 Bochum

www.wtech.rub.de 